\title{
Consecuencias en el diente permanente posterior a traumatismo dentoalveolar de su predecesor diente primario. Reporte de caso
}

Consequences on permanent teeth after a dental trauma to the temporary predecessor: a case report

Consequências sobre os dentes permanentes após um trauma dentário para o predecessor temporário: um relato de caso

\section{Zayda C. Barrios G. ${ }^{1}$ \\ Leiver A. Quintero C. ${ }^{2}$}

Recibido: 6 de junio de 2020

Aprobado: 4 de octubre de 2020

Publicado: 7 de diciembre de 2020

Cómo citar este artículo:

Barrios ZC, Quintero LA. Consecuencias en el diente permanente posterior a traumatismo dentoalveolar de su predecesor diente primario. Reporte de caso. Revista Nacional de Odontología. (2020); 16(2), 1-13. doi: https://doi.org/10.16925/2357-4607.2020.02.11

Reporte de caso. https://doi.org/10.16925/2357-4607.2020.02.11

1 Odontopediatría. Departamento de Odontología Preventiva y Social. Facultad de Odontología. Universidad de Los Andes. Mérida-Venezuela. Edif. Rectorado. Calle 24 entre Av. 2 y 3. Mérida-Venezuela. c/postal: 5101.

Correo electrónico: z.barrios@mail.ula.ve,zaydabarrios@gmail.com

2 Ortodoncia. Departamento de Odontología Preventiva y Social. Facultad de Odontología. Universidad de Los Andes. Mérida-Venezuela. ORCID: https://orcid.org/0000-0001-8839-2384 
2 Consecuencias en el diente permanente posterior a traumatismo dentoalveolar de su predecesor diente primario. Reporte de caso

\title{
Resumen
}

Introducción: Los traumatismos buco dentales observados en pacientes pediátricos son eventos cotidianos, especialmente durante los tres primeros años de vida. Las consecuencias que pueden tener lugar sobre los dientes definitivos pueden ser anomalías de estructura, anomalías morfológicas de corona o raíz, alteraciones en la cronología de la erupción, retenciones, impactaciones o malformaciones del germen permanente. Independientemente del tipo de traumatismo, el seguimiento es fundamental.

Reporte del caso: paciente masculino 9 meses de edad, quien sufre caída por una escalera. El área traumatizada involucró el sector antero inferior: incisivo central inferior derecho primario con luxación lateral, móvil a la palpación y con desplazamiento del tejido gingival circundante. El incisivo central inferior izquierdo primario con movilidad grado 1. Luego de la valoración clínica y radiográfica se decide realizar la exodoncia del incisivo central inferior derecho primario. El paciente no asistió a las citas de control sino hasta los 7 años, 7 meses de edad, observándose en ese momento, el incisivo central inferior derecho permanente con hipoplasia del esmalte y dilaceración de la corona y el incisivo central inferior izquierdo permanente con hipoplasia del esmalte. Se realizaron interconsultas para decidir la acción a seguir.

Conclusión: Un traumatismo buco dental que involucre a la dentición primaria a corta edad, puede acarrear consecuencias para la dentición permanente, lo que hace necesario el informar a los padres de manera comprensible para facilitar la evaluación y el procedimiento odontológico oportuno a seguir.

Palabras clave: dentición primaria, traumatismo dental, consecuencias, anomalías dentarias.

\begin{abstract}
Introduction: Pediatric dental traumas are common events before 3 years old. Among common consequences in permanent teeth successors are structural anomalies, crown or roots morphologic anomalies, eruption anomalies and impacted teeth. Whatever the type of trauma may be, monitoring is important.

Case report: This paper reports a case of dental-alveolar trauma in a 9-month-old boy, who fell down in a pair of stairs. The traumatic area included: right temporary central lower incisor with lateral luxation, mobile and the gum surrounding displacement; left temporary central lower incisor with one grade of mobility. After the clinical and radiological diagnosis, the decision is to extract the right temporary central lower incisor. Patient did not come again to de control appointment until he was around 7-year, 7 months-old. The eruption of the right permanent central lower incisor occurred, which presented crown dilaceration and enamel hypoplasia. The left permanent central lower incisor with enamel hypoplasia. Multidisciplinary appointments were made to decide actions to take with the permanent teeth as a result of trauma to the temporary teeth.
\end{abstract}

Conclusion: Temporary dental trauma at an early age can produce consequences to the permanent successor. It is important to inform parents in order to make easy the evaluation and the opportune dental procedures.

Keywords: temporary dentition, dental trauma, consequences, dental anomalies

\section{Resumo}

Introdução: Traumas dentários pediátricos são eventos comuns antes dos 3 anos de idade. Entre as consequências comuns em sucessores de dentes permanentes estão anomalias estruturais, anomalias morfológicas da coroa ou raízes, anomalias de erupção e dentes retidos. Qualquer que seja o tipo de trauma, o monitoramento é importante.

Relato de caso: Este artigo relata um caso de traumatismo dentário-alveolar em um menino de 9 meses, que caiu em um par de escadas. A área traumática incluiu: incisivo central inferior direito temporário com luxação lateral, móvel e deslocamento ao redor da gengiva; incisivo central inferior esquerdo temporário com um grau 
de mobilidade. Após o diagnóstico clínico e radiológico, a decisão é extrair o incisivo central inferior provisório direito. 0 paciente não voltou à consulta de controle até os 7 anos e 7 meses de idade. Ocorreu erupção do incisivo inferior central permanente direito, que apresentava dilaceração da coroa e hipoplasia do esmalte. 0 incisivo inferior central permanente esquerdo com hipoplasia de esmalte. Foram feitas consultas multidisciplinares para decidir as ações a serem tomadas com os dentes permanentes em decorrência de trauma nos dentes temporários.

Conclusão: 0 trauma dentário temporário em idade precoce pode trazer consequências ao sucessor permanente. É importante informar os pais para facilitar a avaliação e os procedimentos odontológicos oportunos.

Palavras-chave: dentição temporária, trauma dentário, consequências, anomalias dentárias.

\section{Introducción}

Los traumatismos bucodentales frecuentemente observados en pacientes pediátricos, son el resultado de impactos, cuya fuerza agresora, supera la resistencia de los tejidos óseo, muscular y dentario. Estos son eventos cotidianos, especialmente durante los tres primeros años de vida, etapa en la que el niño no tiene los movimientos locomotores bien desarrollados, las reacciones de defensa son aún lentas y una condición de dependencia que lo hace incapaz para evaluar el riesgo de sus actividades. Por lo general, son las urgencias en odontología que causan mayor impacto emocional y psicológico en el paciente niño y en sus padres (1-4).

Las caídas son los traumatismos de mayor prevalencia y cuando ocurren durante la dentición primaria, debe tenerse presente la estrecha relación entre el ápice del diente primario lesionado y el germen dentario permanente subyacente (pues hay una barrera de tejido duro menor de $3 \mathrm{~mm}$ ), esto ocasiona consecuencias graves a largo plazo, que pueden acaecer sobre el propio diente temporal o sobre el germen del diente permanente (1-5).

La lesión puede derivarse de diferentes causas, entre ellas: por impacto directo que ocasiona el ápice del diente primario sobre el germen del diente permanente en desarrollo, por una lesión indirecta a causa de una necrosis pulpar del diente primario con su consecuente infección periapical en la vecindad con las estructuras del órgano del esmalte o cuando se realiza la exodoncia del diente primario con técnicas indicadas para la extracción de dientes permanentes. El traumatismo en dentición primaria puede afectar la corona, la raíz o al germen del diente permanente en su totalidad $(1,6,7)$.

Las alteraciones en los dientes permanentes, secundarias a un traumatismo ocurrido en la dentición primaria, no diagnosticada y no tratada correctamente a tiempo, varían entre el 12 y el 69\%. Se estima que aproximadamente el 10\% de las alteraciones de esmalte que afectan al sector anterior están relacionadas con lesiones 
4 Consecuencias en el diente permanente posterior a traumatismo dentoalveolar de su predecesor diente primario. Reporte de caso

en los dientes primarios, aunque la mayoría de las veces haya que esperar hasta que erupcione la dentición definitiva para poder comprobar clínica y radiográficamente si muestra secuelas del acontecimiento $(1,5,6)$.

Entre las consecuencias que pueden tener lugar sobre los dientes definitivos cabe destacar anomalías morfológicas, de estructura, que pueden afectar a la corona (hipoplasia del esmalte, hipocalcificación, dilaceraciones de la corona), raíz (duplicación y dilaceraciones parciales o totales de la raíz) o producir asimismo alteraciones en la cronología de la erupción, retenciones, impactaciones o malformaciones del germen permanente (1-3,5,8).

La manifestación clínica de la lesión dependerá del grado de la fase de la odontogénesis en que se encuentre el permanente en ese momento. Muchos de los accidentes dentoalveolares en el niño se producen con mayor frecuencia entre las edades de uno y medio, a dos y medio, y entre los cuatro y los seis años, observándose mayores complicaciones por traumatismos ocurridos antes de los 4 años de edad. El 63\% de los casos en niños menores de 2 años y el 53\% en niños de 3-4 años, en comparación con los de niños de más edad (1-3,6).

Según el protocolo para el manejo de los traumatismos en dentición primaria de la SEOP (Sociedad Española de Odontopediatría) y la IADT (International Association of Dental Traumatology), si el ápice se ha desplazado hacia el germen del diente sucesor permanente, el diente debe ser extraído, de lo contrario queda invadiendo el espacio ocupado por el germen de reemplazo afectando extensas áreas de la matriz del esmalte en desarrollo o causar un desvío o desplazamiento del germen del permanente y esto puede provocar una disrupción de la vaina epitelial de Hertwig. Además, la recolocación del diente podría causar alteraciones en el germen del diente permanente y su inmovilización a corta edad muchas veces no se hace factible (1,3-5).

Independientemente del tipo de traumatismo, el acompañamiento clínico y radiográfico de los dientes primarios traumatizados es fundamental para identificar tempranamente posibles secuelas que afecten al diente sucesor permanente en desarrollo y así instituir un tratamiento inmediato. No obstante, las citas de control pueden variar según el tipo de traumatismo y observación o no de complicaciones $(8,9)$.

El propósito del presente artículo es señalar el tratamiento realizado ante un traumatismo ocurrido en la dentición primaria y la afección posterior que tuvo lugar en la estructura dentaria del sucesor permanente. 


\section{Caso clínico}

Paciente masculino de 9 meses de edad, procedente del municipio Campo Elías del estado Mérida-Venezuela, que acude con su madre a la consulta odontológica, 24 horas después de haber sufrido un traumatismo buco dental tras caída por escalera en su casa, mientras se movilizaba con una andadera.

A la anamnesis, su madre no hace referencia a antecedentes médicos en el niño, ni familiares relevantes. Manifiesta haber asistido al pediatra inmediatamente después del incidente para su valoración correspondiente, sin embargo, no se presentaron signos o síntomas que obligaran su hospitalización, ni ameritó medicación alguna, solo vigilancia y la necesidad de consultar lo más pronto posible odontopediatría.

Una vez que se obtiene la autorización de la madre, previa información con relación al carácter inocuo de la inspección a realizar, se procedió con la exploración extraoral, donde no se observaron lesiones en los tejidos circundantes a la cavidad bucal que ameritasen tratamiento. A la exploración intraoral, se encontraron presentes solo los incisivos centrales y laterales superiores e inferiores derecho e izquierdo primarios.

La zona traumatizada involucró el sector antero inferior, observándose que el incisivo central inferior derecho primario o Unidad Dentaria 81 (UD81) había sufrido una luxación lateral, encontrándose móvil a la palpación y con desplazamiento también del tejido gingival circundante. El incisivo central inferior izquierdo primario (UD71) estaba con movilidad grado 1. El resto de las estructuras blandas estaban sin erosiones o abrasiones (figura 1).

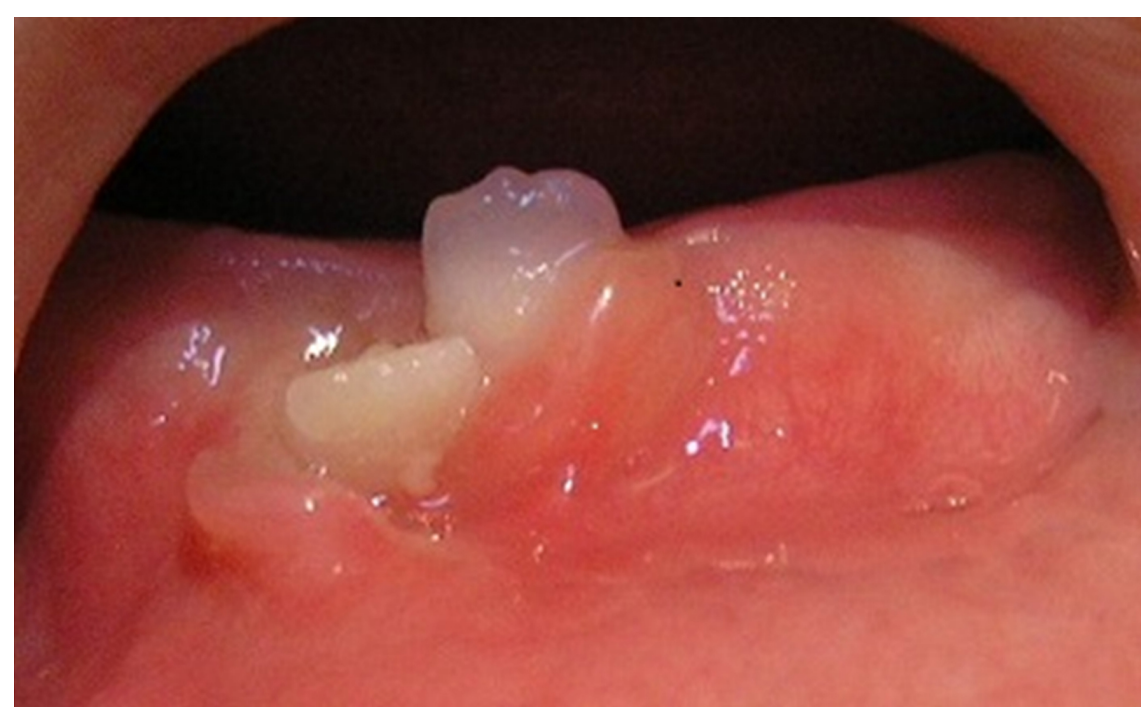

Figura 1. Fotografía que muestra al incisivo central inferior derecho primario con luxación lateral

Fuente: elaboración propia. 
Después, se procedió a realizar una radiografía extraoral lateral utilizando para ello una película radiográfica oclusal para ayudar en la determinación de la posición de la raíz de la pieza dentaria desplazada. La imagen radiográfica mostró el desplazamiento vestibular de la corona de la UD81 y el desvío del ápice dentario hacia la tabla lingual, aparentemente involucrando los tejidos que corresponden a los gérmenes de reemplazo de los incisivos inferiores. Esta evaluación radiográfica confirmaba el diagnostico de luxación lateral (figura 2).

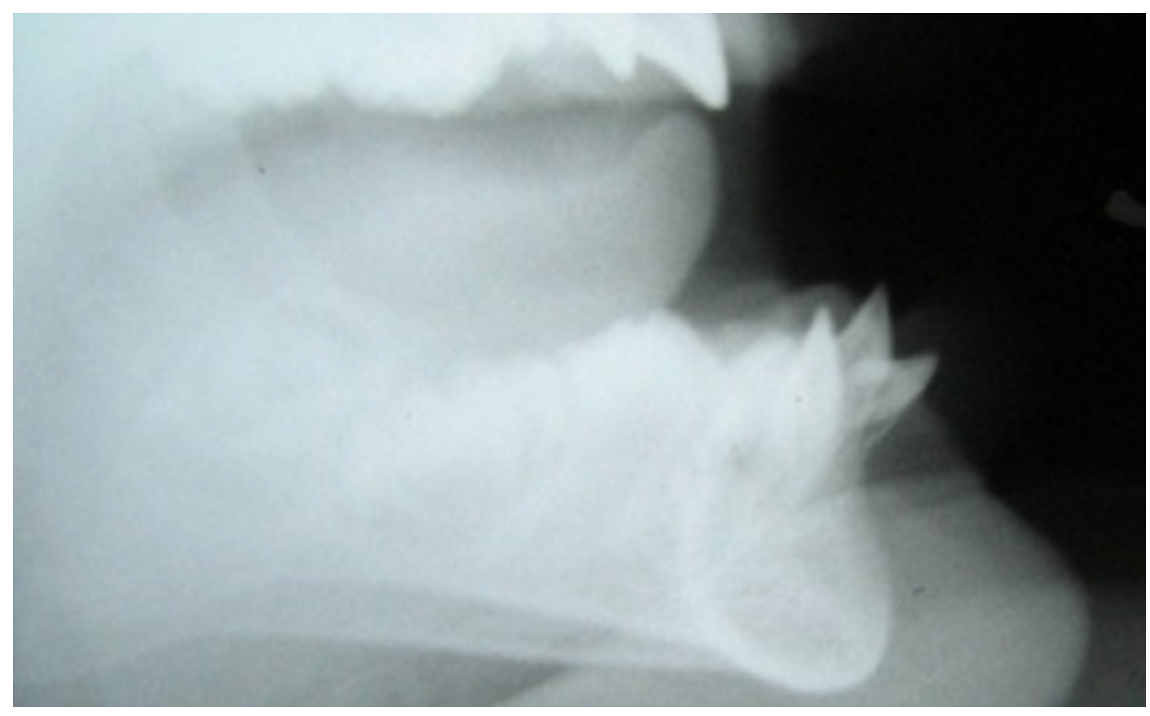

Figura 2. Imagen radiográfica lateral que muestra la luxación lateral del Incisivo central inferior derecho primario

Fuente: elaboración propia

Nuevamente se solicita a la madre el consentimiento informado (manejo de conducta, sus beneficios y posibles complicaciones) para realizar el tratamiento odontológico requerido para el niño: la exodoncia del incisivo central inferior derecho primario bajo anestesia local (antisepsia, anestesia tópica y Lidocaina al 2\% (Rapicaine R)); la misma se realizó sin complicaciones. No se indicó tratamiento alguno, salvo los controles periódicos correspondientes para un traumatismo, sin embargo, a pesar de la información e indicaciones dadas a la madre, no fue posible realizar las revisiones programadas para comprobar la curación y la erupción de los incisivos centrales permanentes.

El paciente acude nuevamente a consulta a los 7 años, 7 meses de edad. La madre manifiesta no haber cumplido con las citas de control posteriores al traumatismo debido a descuido de su parte, a pesar de las indicaciones dadas anteriormente por parte del profesional. La madre y el paciente deciden volver a consultar debido al 
color y a la posición que presentan las piezas dentarias permanentes en erupción y al antecedente de traumatismo sufrido en esa misma área, a corta edad.

No refieren antecedentes médicos personales o familiares de importancia. A la valoración intraoral se observó: incisivo central inferior derecho permanente (UD41) con hipoplasia del esmalte y dilaceración de la corona, incisivo central inferior izquierdo permanente (UD31) con hipoplasia del esmalte (figuras 3, 4 y 5).
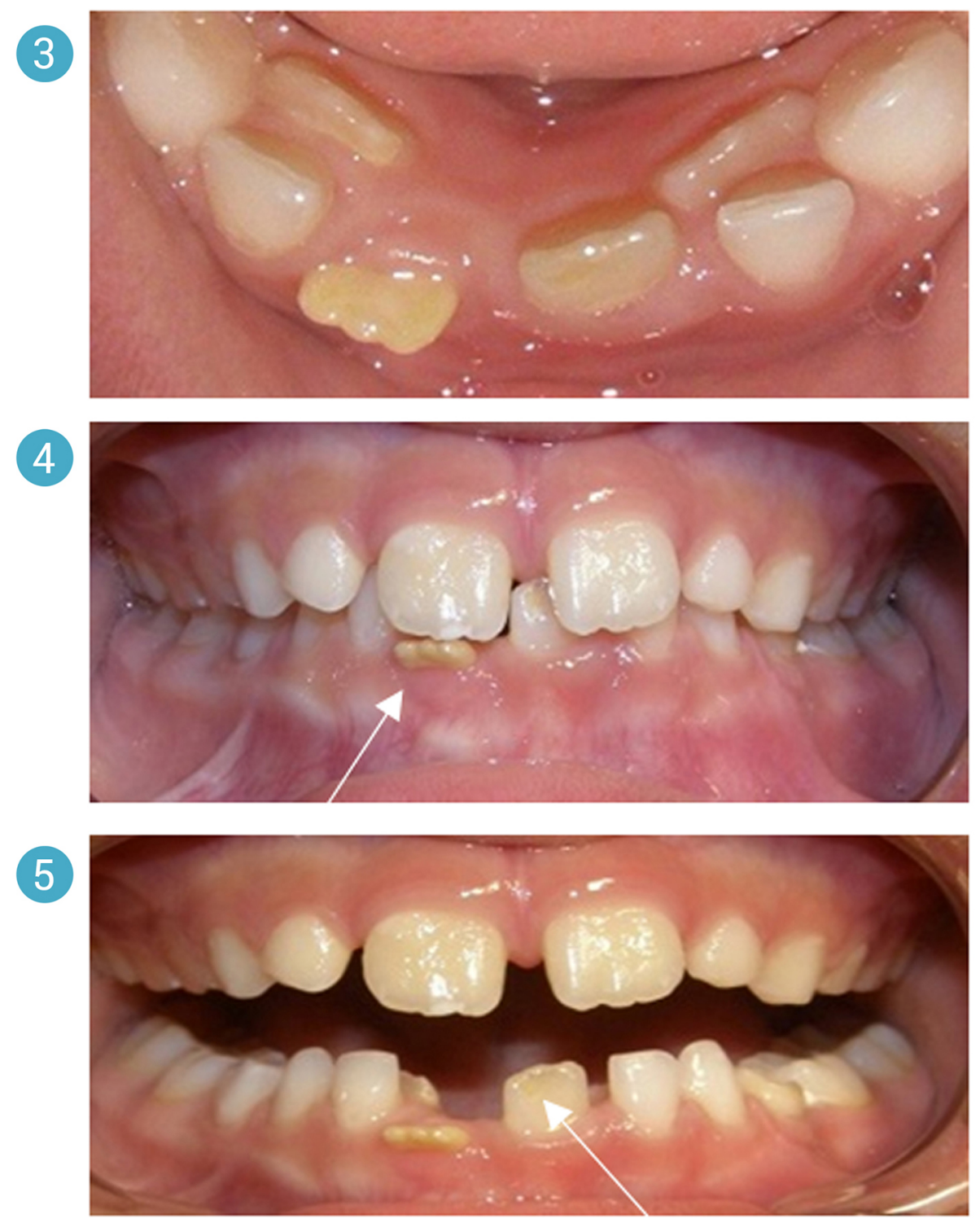

Figuras 3, 4 y 5. Fotografías mostrando los defectos de forma y estructura en los incisivos centrales inferiores derecho e izquierdo permanentes

Fuente: elaboración propia. 
8 Consecuencias en el diente permanente posterior a traumatismo dentoalveolar de su predecesor diente primario. Reporte de caso

Al paciente se le realizó un control radiográfico para determinar el desarrollo radicular de ambos incisivos. La imagen radiográfica mostró una clara constricción de la corona (dilaceración) de la UD41 e hipoplasia de esmalte en la misma unidad dentaria; en la UD31, área hipoplásica de esmalte. No se apreciaron alteraciones a nivel radicular y se corroboró la salud pulpar, no ameritando por el momento tratamiento de endodoncia (figura 6).

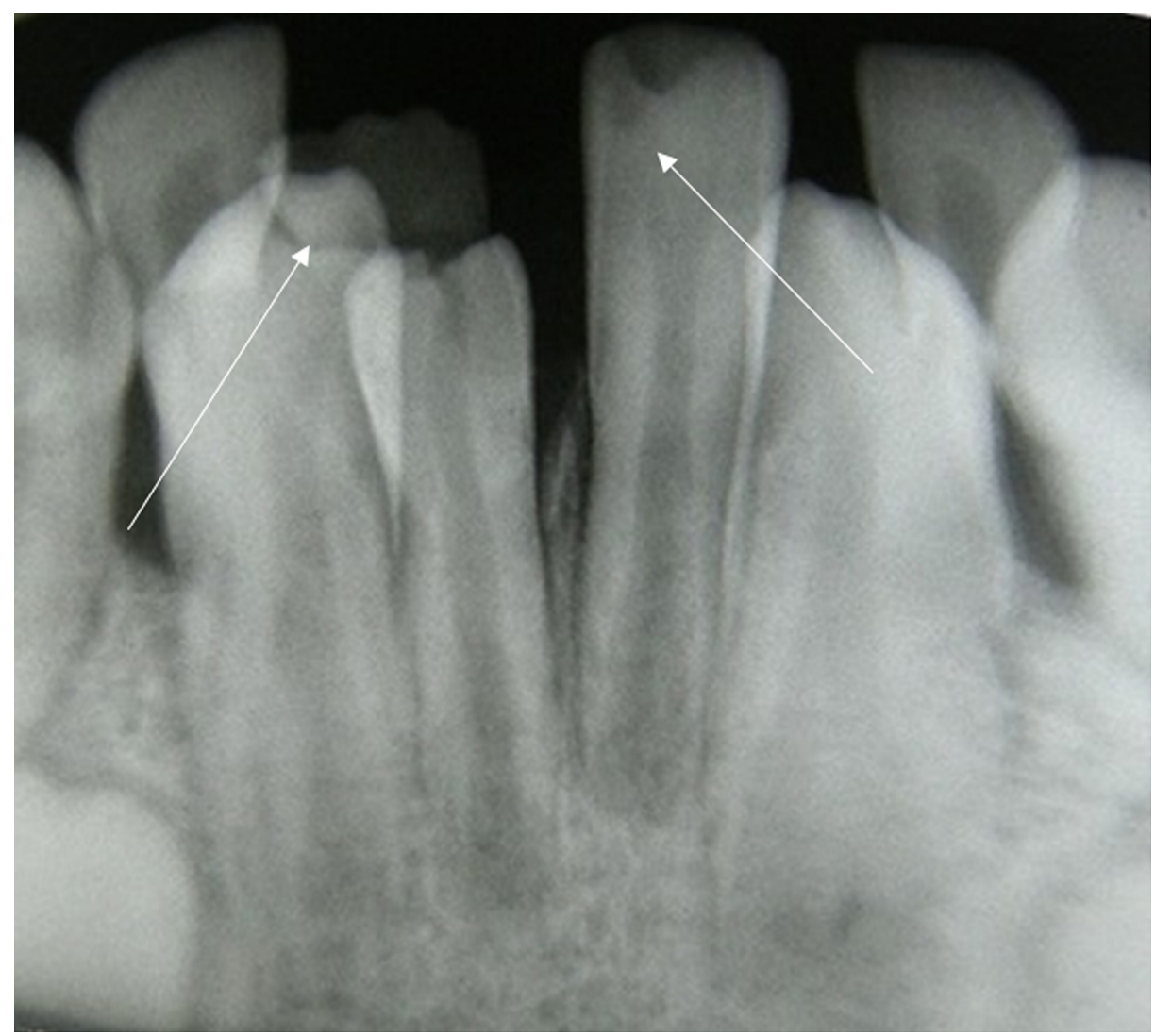

Figura 6. Imagen radiográfica mostrando (flecha color blanca) los defectos de forma y estructura en los sucesores permanentes Fuente: elaboración propia.

Al paciente se le realizaron interconsultas con áreas asociadas a la odontopediatría para decidir la acción a seguir con los incisivos permanentes inferiores, involucrados en el traumatismo buco dental y se programaron nuevamente citas de control clínico y radiográfico en la medida que hacían erupción las piezas dentarias del área en observación. 


\section{Discusión}

Tras todo proceso traumático en la dentición primaria es preciso considerar que, las raíces de los incisivos primarios inferiores se hallan normalmente por labial de los gérmenes dentales de los correspondientes incisivos sucedáneos en desarrollo y en razón de la conformación ósea de los maxilares, es más probable que un diente primario afectado por un impacto traumático sea desplazado y no fracturado (2).

Hernández et al. (6) determinaron que en un 20,9\%, la luxación lateral era uno de los diagnósticos prevalentes luego de un traumatismo dentoalveolar en la dentición primara y la frecuencia de secuelas en dientes permanentes por afección en la dentición primaria de un 58\%. Pérez (3) y Silva (2) concuerdan al describir una frecuencia más alta de defectos del esmalte tras una lesión con desplazamiento en comparación con lesiones sin desplazamiento. Al moverse la raíz en dirección palatina o lingual puede causar lesiones leves como la hipoplasia de esmalte y movimientos menores en su trayecto eruptivo o también es factible que se produzcan daños mayores del diente permanente después de la incompleta o el total progreso de la corona (2).

La hipoplasia ocurre cuando el trauma afecta antes de la mineralización afectando a la matriz y el resultado es la destrucción de los ameloblastos activos, es decir, hay falta de esmalte, y aparecen fosetas, surcos, estrías y cavitaciones con decoloraciones blancas o marrón amarillentas. Radiográficamente, como no hay esmalte, puede evidenciarse áreas radiolúcidas en la porción coronaria, justo donde hubo fallas en la deposición de la matriz $(7,10)$.

Lo descrito anteriormente se corresponde con las lesiones que aparecen en el caso reportado. El incisivo central inferior derecho primario (UD81) sufrió una luxación lateral, lo que a la larga repercutió en el germen de reemplazo. Una vez que este hizo su erupción en boca, se pone en evidencia el trastorno: hipoplasia de esmalte en la corona de la UD 41, incluso área hipoplásica en vestibular de la UD 31.

La dilaceración de la corona es otro tipo de defecto que se caracteriza por una desviación aguda del eje longitudinal de la corona que puede deberse al desplazamiento no axial del tejido duro ya formado, en relación con el tejido en desarrollo no calcificado. Después de la invasión al folículo, el epitelio del esmalte desplazado se activa en la nueva posición, dando lugar a una nueva forma de esmalte que se proyecta en el interior del conducto o externamente en la unión de la corona-raíz. Radiográficamente, la corona dilacerada presenta una imagen deformada y escorzada en relación al aspecto normal de los dientes adyacentes. La erupción puede ocurrir normalmente $(11,12)$.

Pérez (3) y Mellara et al. (12) coinciden en señalar que la dilaceración de corona ocurre con menos prevalencia y de presentarse con angulación labial, es más común 
en los incisivos inferiores. En el caso clínico que aquí se describe, aparece una dilaceración de la corona de la UD 41 y corresponde con lo descrito anteriormente, la pieza dentaria hizo erupción sin dificultad y la angulación labial se observa en el incisivo central inferior permanente.

La dilaceración coronaria tiende a desarrollar una necrosis pulpar seguida de una lesión apical. Para evitar estas complicaciones se sugiere remover la porción coronaria después de su erupción y colocar una corona provisional hasta que se confeccione una restauración definitiva (12), sin embargo, en este caso, no existía patología pulpar que ameritara su tratamiento.

La corona del diente permanente es la zona más afectada, y a edades en las que esta se está formando; entre los estadios de Nolla 0 y 6, es decir, entre los primeros meses de vida y los 4 años. Además, podría deberse a que la reabsorción fisiológica de la raíz genéticamente programado cuando el niño tiene aproximadamente 52 meses de edad. En los pacientes de mayor edad, un traumatismo en el diente primario no produciría el mismo daño en el germen permanente $(2,3,6,10)$.

El sexo masculino es el más afectado cuando se valoran secuelas en la dentición permanente por traumatismos en la dentición primaria, debido a que los niños son más enérgicos y activos entre los 1 y 4 años de edad y el lugar preferencial para la ocurrencia traumática dental en los niños de temprana edad, es el propio hogar $(2,4,6,10)$. El caso reportado describe el accidente ocurrió en un paciente de sexo masculino a los 9 meses de edad, en el que se ven afectados los dientes permanentes una vez que hicieron su correspondiente erupción en cavidad bucal.

Los tratamientos requeridos en caso de trauma bucodental en pacientes de corta edad tienen sus limitaciones: la cantidad de dientes en boca y la inmadurez psicológica hacen que casi siempre no se puedan ejecutar las terapéuticas deseadas e incluso necesarias. Cuantos menos dientes tenga, más difícil será de hacer la inmovilización de algún diente con movilidad o que haya sido recolocado en su lugar (4). En el caso clínico que se reporta, la corta edad del paciente, su poca colaboración a la evaluación, el número de piezas dentarias presentes en el área afectada, indica que la exodoncia de la UD81 es la terapéutica a seguir. Además, es imposible la recolocación del diente y su ferulización, así como es factible, que se produzcan daños mayores en los dientes permanentes aún no presentes en cavidad bucal.

La detección temprana de esas alteraciones posibilita el tratamiento adecuado. Por tal motivo, se recomienda que después de la atención inicial, el paciente vuelva a la consulta para la evaluación clínica y radiográfica correspondiente después de 30 y 90 días, 5, 8 y 12 meses y luego cada 6 meses hasta la erupción del sucesor permanente. El tratamiento a seguir muchas veces es multidisciplinario (ortodoncia, 
cirugía, endodoncia, prostodoncia) dependiendo de la extensión y la severidad de las secuelas $(7,12)$.

Una limitación que se presentó en este caso fue lo difícil del seguimiento durante el tiempo transcurrido entre el traumatismo del diente primario y la erupción del diente permanente, el niño fue valorado nuevamente a los 7 años y 7 meses de edad y la madre reconoció su descuido al respecto. Se coincide con Nogueira et al., (4) quienes señalan que el grupo de niños con edades inferior a cuatro años, no son llevados a la atención correspondiente.

Pérez (3) señala que los padres manifiestan inicialmente mucha preocupación con la severidad del trauma, la cantidad de dientes lesionados y el tipo de dentición afectada. Pero esta preocupación disminuye con el tiempo y dificulta la asistencia a los controles posteriores. También el dolor que pueda ocasionarse en la cita inicial distancia el tratamiento dental más adelante y se hace difícil convencerlos de que regresen a la consulta de seguimiento (6).

Otro hallazgo encontrado en el presente caso fue la erupción ectópica de la UD32 y UD42. Según Pérez (3) y Maldonado (13) esto puede ser a consecuencia de un traumatismo en la dentición primaria que causa el desplazamiento físico y cambia la trayectoria de erupción del germen del diente permanente, convirtiéndose en un factor predisponente de una malposición dentaria.

En el caso que se reporta, la ausencia de complicaciones (dificultad en la erupción, patología pulpar, baja autoestima) que ameritaran de tratamientos de urgencia permitió la interconsulta correspondiente con ortodoncia y con odontología restauradora para proceder con la secuencia y con el tratamiento pertinente cuando correspondiera.

\section{Conclusión}

Un traumatismo bucodental como la luxación lateral que involucre a la dentición primaria a muy corta edad acarrea consecuencia para la dentición permanente como son los defectos de forma y de estructura. Por ello, se precisa informar a los padres de manera comprensible para facilitar la evaluación y el procedimiento odontológico oportuno. 
12 Consecuencias en el diente permanente posterior a traumatismo dentoalveolar de su predecesor diente primario. Reporte de caso

\section{Referencias}

1. Barberia E; Borrel C; Bravo N; Cardoso C; Maroto M. Traumatismos en los dientes temporales: ¿causan secuelas en los dientes permanentes? Gaceta Dental. 2010; 210, 124-137.

2. Silva-Reggiardo E. Secuelas en la dentición permanente por traumatismo dentoalveolar en dentición decidua. Reporte de caso. Odontol Pediatr. 2012; 11(1): 57-63.

3. Pérez de Mora E. Traumatismos en dentición primaria. Secuelas postraumáticas en dentición permanente. Revisión sistemática. [Tesis de grado] Sevilla (E): Universidad de Sevilla; 2018.

4. Nogueira AJSN; Nogueira RGM; Corrêa DB; Souza FX. Traumatismo dental en bebés. Artículo de revisión. Rev de Odontopediatría Latinoamericana. 2018; 8(1): 66-74.

5. Lacalle C; Muñoz M. Luxación intrusiva de un incisivo central superior temporal con perforación de la tabla vestibular. Presentación de caso clínico. RCOE. 2019; 24(4): 118-122.

6. Cueto A; Rivera C; González J. Traumatismos Dentoalveolares que Afectan a las Estructuras de soporte de los dientes temporales y sus efectos en los sucesores definitivos. Int. J. Odontostomat. 2012; 6(3):379-383.

7. Hernández MJ; Vilches NF. Secuelas en dentición permanente a partir de trauma dentoalveolar en dentición temporal, en pacientes atendidos en el sub Departamento de Odontología del Hospital Base Valdivia-Chile. [Tesis de grado] Valdivia (C): Universidad Austral de Chile; 2017.

8. Nelson-Filho P; Assed S; Bezerra da Silva LA. Traumatismos en la dentición temporal. En: Bezerra da Silva LA. Tratado de Odontopediatría. Tomo 2. Colombia: AMOLCA; 2008. p. 811-855.

9. Carvalho P. Aspectos radiográficos de imágenes periapicales asociadas a incisivos primarios traumatizados. Odontología. 2018; 20(1): 136-146.

10. Bardellini E, Amadori F, Pasini S, Majorana A. Dental Anomalies in Permanent Teeth after Trauma in Primary Dentition. J Clin Pediatr Dent. 2017; 41(1): 5-9.

11. Mendoza A, Iglesias A, Yañez R, Abalos C. Prevalence and complications of trauma to the primary dentition in a subpopulation of Spanish children in southern Europe. Dent Traumatol. 2015;31(2):144-149. 
12. Mellara TS, Nelson-Filho P, Queiroz AM, Santamaria Júnior M, Bezerra da Silva RA, Bezerra da Silva LA. Crown Dilaceration in Permanent Teeth after Trauma to the Primary Predecessors: Report of Three Cases. Braz Dent J. 2012; 23(5): 591-596.

13. Maldonado-Villamizar J. Manejo ortodóntico-interceptivo para trastornos eruptivos de incisivos centrales permanentes como consecuencia de un trauma dentoalveolar en dentición primaria. Reporte de caso. Rev Venez Invest Odont IADR. 2016; 4(1): 83-91. 